Ezio Fanucci

Salvatore Masala

Sebastiano Fabiano

Dario Perugia

Ettore Squillaci

Viviana Varrucciu

Giovanni Simonetti

\section{Treatment of intermetatarsal Morton's neuroma with alcohol injection under US guide: 10-month follow-up}

Received: 19 December 2002

Revised: 2 June 2003

Accepted: 14 July 2003

Published online: 3 October 2003

(C) Springer-Verlag 2003

E. Fanucci $(\varangle) \cdot$ S. Masala $\cdot$ S. Fabiano E. Squillaci - V. Varrucciu · G. Simonetti Department of Diagnostic Imaging and Interventional Radiology,

University of Rome "Tor Vergata", Viale Oxford 81, 00133 Rome, Italy

e-mail: sebas575@yahoo.it

Tel.: +39-6-20902401

Fax: +39-6-20902404

D. Perugia

Department of Orthopaedic Surgery, University of Rome "Tor Vergata",

Viale Oxford 81, 00133 Rome, Italy

\begin{abstract}
Morton's neuroma (MN) is a frequent cause of metatarsalgia. The aim of our study was to evaluate the efficacy of neuroma alcoholsclerosing therapy (NAST) under US guide in MN after a 10-month follow-up. Forty intermetatarsal neuromas underwent alcohol-sclerosing therapy after sonographic evaluation of their dimensions and echotexture. After subcutaneous anesthesia, a sclerosing solution composed of anesthetic (carbocaine-adrenaline 70\%) and ethylic alcohol (30\%) was injected inside the mass under US guidance. The procedure was repeated at intervals of 15 days until the resolution of the symptoms. A total or partial symptomatic relief was obtained in 36 cases $(90 \%)$. No procedure-related
\end{abstract}

complications were observed. Transitory plantar pain, due to the flogistic reaction induced by the sclerosing solution, occurred in 6 cases $(15 \%)$. The 10 -month follow-up revealed a $20-30 \%$ mass volume reduction and an adiposus-like change in echotexture. In the 4 cases $(10 \%)$ of therapeutic failure, the preliminary sonography demonstrated a hypoechoic echotexture with a strong US beam attenuation corresponding to a highly fibrous neuroma after surgical resection. The NAST is a feasible and cost-efficient procedure with high rates of therapeutic success.

Keywords Foot Alcohol . Morton's neuroma · US · Peripheral neuropathies

\section{Introduction}

Plantar interdigital neuroma, better known as MN, is a common disorder of the foot and is associated with forefoot pain and paresthesias caused by perineural fibrosis $[1,2]$.

This condition was first described by Civinini in 1835, Durlacher 1845 [3], and subsequently by Morton in 1876 [4].

The etiology is unknown. Many authors believe that an excessively pronated foot and cavus feet are usually implicated because of the increased tension on the plantar fascia and the intermetatarsal ligament $[5,6,7,8]$.

The lesion consists of perineural fibrosis, local vascular proliferation, edema of the endoneurium, and axonal degeneration. Histologically, this condition is caused by fibrous degeneration of the soft tissues around the nerve, demyelination, and endoneurial fibrosis of the nerve [9, $10,11,12,13,14]$.

The diagnosis may be made through the clinical history of pain radiating from the mid-foot to the toes on sonography and MRI $[8,9,15,16,17]$.

There exist various conservative and surgical methods of treatment for interdigital neuroma [18, 19, 20, 21, 22, $23,24,25,26,27,28]$.

Dockery [21] in 1999 tried a new therapeutic approach based on the injection of a $4 \%$ alcohol solution inside the lesion. Another group tested this possibility increasing the concentration of alcohol from 4 to $30 \%$ and reducing the number of injections. Moreover, this group was the first to engage a sonographic guide for the puncture of the neuroma [21,22]. 
The aim of this study was to evaluate NAST as a viable alternative to steroid injections or surgery in the treatment of MN associated with persistent symptoms.

\section{Materials and methods}

From January 1999 to June 2001, 40 consecutive patients (7 men and 33 women with a mean age of 48 years, range $28-65$ years) with interdigital neuroma underwent NAST using a 30\% alcoholsclerosing solution.

Patients with previous surgical therapy or other associated foot problems were excluded from the study.

All patients typically complained of pain and numbness in the affected interspace, exacerbated by tight-fitting footwear, and most complained of the sensation of a small plantar bump in the same site. All patients used orthotic devices before treatment without benefits. Twenty-two MNs involved the right foot, whereas 18 were located in the left foot. In all cases the neuroma was localized in the third interdigital space. The clinical diagnosis obtained through Mulder's maneuver was confirmed by US performed using 7.5- and $10-\mathrm{MHz}$ linear-array transducers. Using a plantar and a dorsal approach, the patients were scanned in the transverse and longitudinal planes with regard to the metatarsal shafts. Thereafter, the longitudinal and transverse diameters of the neuroma were measured $[14,29]$.

Informed consent was obtained in all patients prior to the procedure. Each patient was positioned supine with their knee flexed at $45^{\circ}$, with a hard pillow placed under the plantar surface of the foot in order to raise the metatarsophalangeal area.

Using a dorsal approach, a 26-G needle was positioned under sonographic guidance in the intermetatarsal space cranially to the

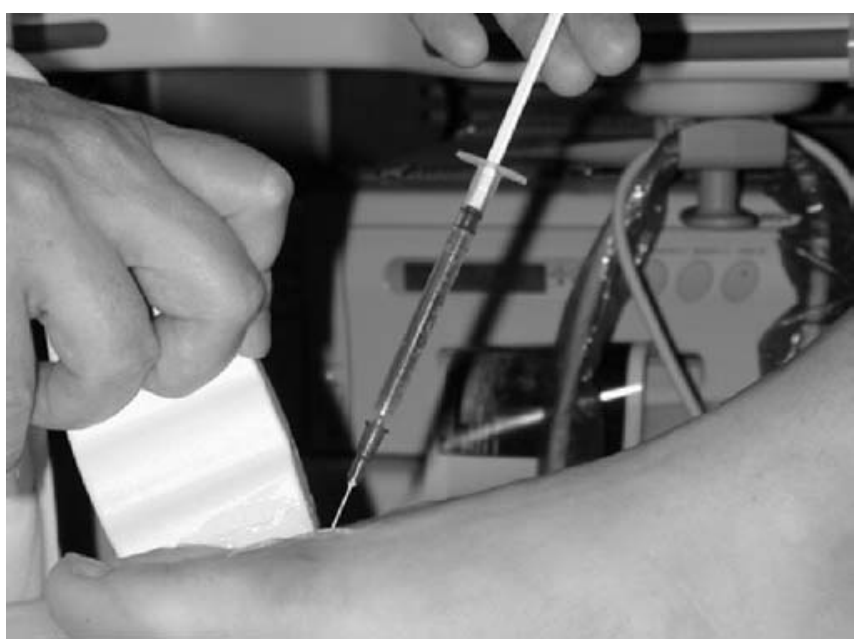

Fig. 1 Technique of neuroma alcohol-sclerosing therapy (NAST): dorsal approach, craniocaudal needle direction head of the neuroma. After subcutaneous injection of $0.5-1 \mathrm{ml}$ carbocaine $2 \%$ solution, the injection of $0.5 \mathrm{ml}$ sclerosing solution composed of $70 \%$ carbocaine and $30 \%$ ethylic alcohol $95 \%$ was performed through a 22-G needle (Fig. 1). The solution was slowly injected into the neuroma following the liquid flow inside the tissue. This procedure enables one to stop injecting whenever a leakage of the liquid is seen inside the plantar fat, which is perceived by the patient as a strong pain. At the end of treatment a US control scan was performed in order to exclude complications. Each subject underwent four injections at 15-day time intervals in order to avoid strong flogistic reactions.

Therapeutic success was established at the short-term followup (15 days) only on the basis of the remission of symptoms. The mass echotexture changes and the volume reduction were considered in the long-term follow up (10 months).

\section{Follow-up}

A clinical and sonographic follow-up was performed 10 months after the last injection. At the follow-up, patients were asked to grade the outcome of their treatment program using the system advocated by Johnson et al. (Table 1) [30]:

1. Completely satisfied (essentially pain free, no restrictions in activity, and only minor restriction in footwear)

2. Satisfied with minor reservations (occasional mild pain, minor restrictions in activity, and minor restrictions in footwear)

3. Satisfied with major reservation (mild or moderate pain, moderate restrictions in activity and major restrictions in footwear, but overall improvement)

4. Dissatisfied (no improvement in pain or worse pain, major restrictions in activity and footwear, no improvement or worse symptoms)

Sonographic follow-up was performed to evaluate the variations in shape, diameter, and echotexture of the neuromas.

To evaluate the effects of the procedure, a preliminary scale of sonographic appearance of the neuroma was made choosing as referential parameters the degree of echogenicity of the mass and the attenuation values of the sonographic beam determined by the mass.

Three different types of mass echotexture were found: type A, anechoic mass with low-beam attenuation; type B, hypoechoic mass with intermediate beam attenuation; type $\mathrm{C}$, hypoechoic mass with high-beam attenuation (Fig. 2).

\section{Results}

The preliminary US evaluation revealed a mass echotexture type A in 15 lesions, type B in 21 lesions, and type $\mathrm{C}$ in 4 lesions. The 4 patients with type-C echotexture neuroma underwent surgery (Fig. 2).

A total of 160 injections were performed in 40 patients. Technical success was achieved in all cases (100\%).

Table 1 Johnson's scale

Completely satisfied

Satisfied with minor reservations

Satisfied with major reservation

Dissatisfied
Pain free; no restrictions in activity; only minor restriction in footwear

Occasional mild pain; minor restrictions in activity; minor restrictions in footwear

Mild or moderate pain, moderate; restrictions in activity; major restrictions in footwear but overall improvement

No improvement in pain; worse pain; major restrictions in activity and footwear; no improvement; worse symptoms 

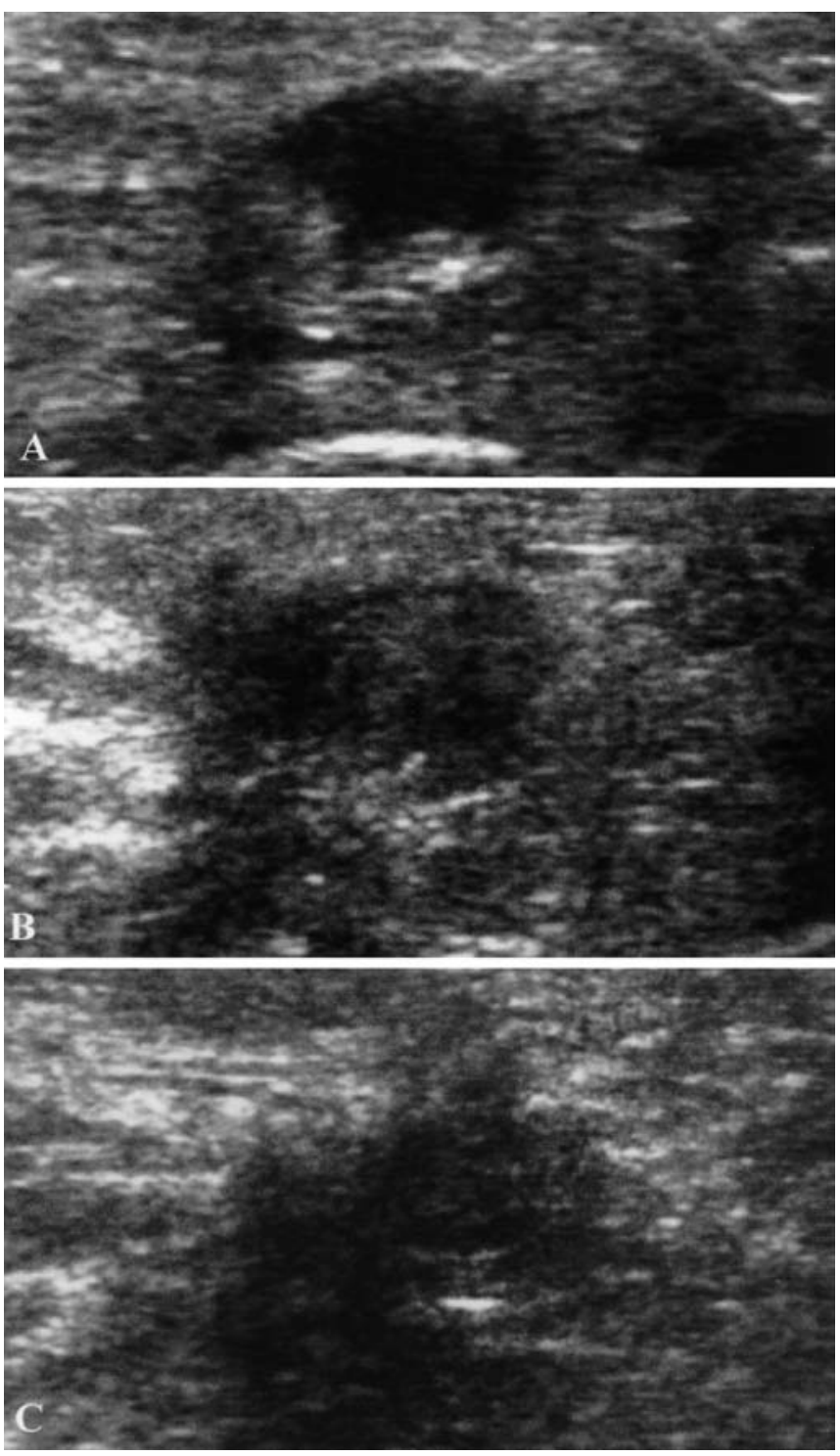

Fig. 2A-C Different types of echotextures. A Anechoic mass and low-beam attenuation. B Hypoechoic mass and medium beam attenuation. C Hypoechoic mass and high-beam attenuation

A moderate pain in the site of the anesthesia was referred after 23 injections disappearing after 3-4 days in all cases.

A transitory plantar pain due to a subtle leakage of fluid was referred after 12 injections. No major procedure-related complications occurred.

At the short-term 15 days follow-up from the last injection a partial (17 patients; $42.5 \%)$ or total (19 patients; $47.5 \%$ ) relief of sensory disturbances and pain was observed in 36 cases $(90 \%)$. Four cases (10\%) where no symptomatic improvement was obtained had to undergo neurectomy and were considered failures of NAST.
The clinical evaluation of the 10-month long-term follow-up was performed according to Johnson et al.'s [30] scale: 21 patients were completely satisfied; 9 resulted satisfied with minor complications; 6 were satisfied with major complications; and 4 patients were dissatisfied and underwent surgery.

Two of the 17 patients with a partial relief of symptoms at the short-term follow-up referred complete satisfaction after 10 months.

The long-term US evaluation considered the changes in mass echotexture and volume (visual evaluation; Fig. 3). All the masses presented an increase in echotexture, with no volume reduction in 15 cases (Fig. 3A) and with volume reduction in 21 cases (Fig. 3B). The echotextural classification of neuromas is carried out only in the preprocedural phase to obtain a prognostic factor of the procedural success. This evaluation is not applicable after alcoholization.

Mass volume reduction was considered as a reduction of at least $20 \%$ in the longitudinal or transverse diameter. All the 21 patients with a positive Johnson et al. [30] scale for complete satisfaction presented a mass volume reduction.

\section{Discussion}

Morton's neuroma is as Morton described it in 1876: "a particular and painful affection of the fourth metatarsophalangeal articulation". This disease is one of the most common disorders of the foot [2]. The differential diagnosis of MN must be made with stress fracture, tendon sheath ganglion, foreign body reaction, nerve sheath tumor, dislocation of the metatarsophalangeal joint and intermetatarsal bursitis [15]. Diagnosis of $\mathrm{MN}$ is usually made through an accurate anamnesis and physical examination and must be confirmed by US [9].

Sonography is considered as an accurate means of revealing MN. Several studies identified $95-98 \%$ of MNs prospectively and up to $100 \%$ retrospectively [9]. Neuromas have a US appearance of a hypoechoic mass, with regard to the muscle. Many studies emphasize the importance of US imaging in the establishment of the presence of multiple neuromas, the exact dimensions, and localization $[9,16,17]$. Shapiro and Shapiro described hypoechoic areas within some neuromas and proposed that these were correlated to the adipous envelopment of the mass [17]. Some authors advocate MRI with good results in terms of sensitivity and specificity [15]. Although the cause remains uncertain, in most cases a treatment is mandatory due to limitations in the patient's activities.

The injection of corticosteroids associated to an anesthetic into the affected interspace is a widely acknowledged technique. More recently, the decompres- 


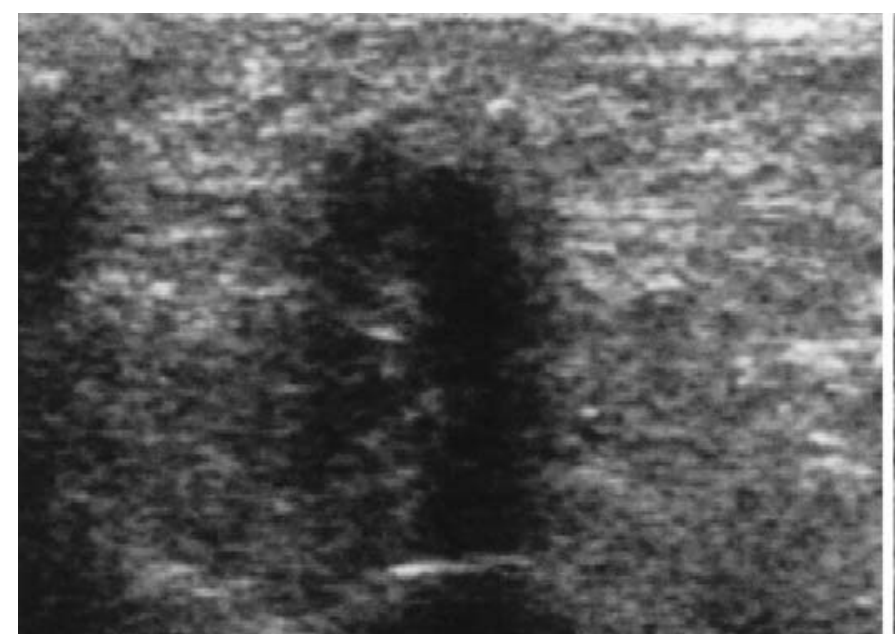

\section{A Pre NAST}

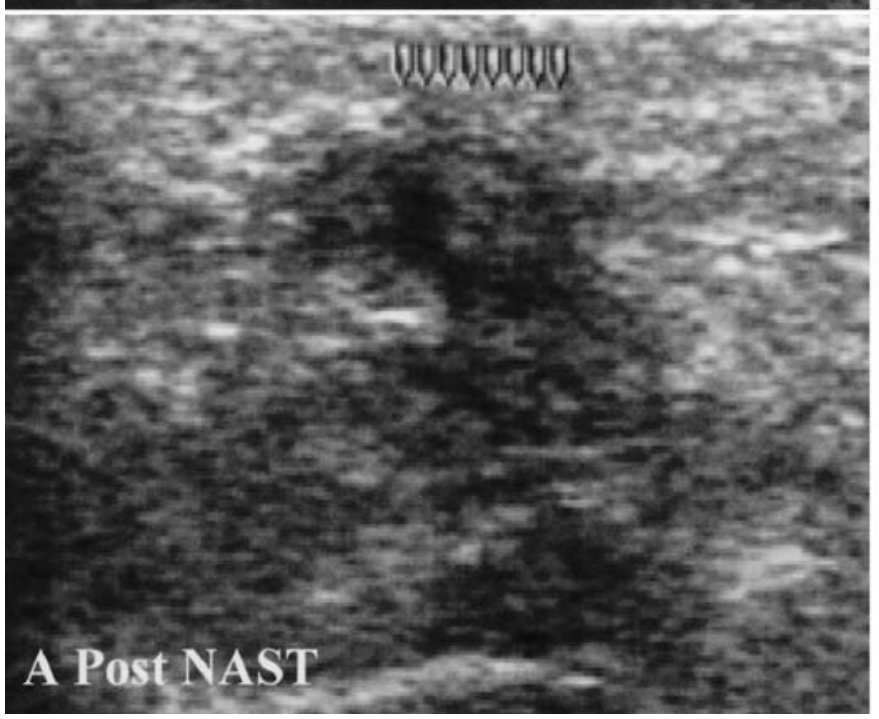

Fig. 3 Two lesions before NAST and after 10-month follow-up

sion of the involved interdigital nerve by the division of the transverse metatarsal ligament and liberation of the entrapped nerve has reported good results [31].

Sclerosing therapy is a new choice for the treatment of MN. Ethylic alcohol has a great affinity to the nervous tissue and when injected in proximity to the nerve is able to induce dehydration, necrosis, and precipitation of the cell protoplasm resulting in a neuritis with a wallerian nerve degeneration (chemical neurolysis) $[16,21,22$, 32]. Alcohol inhibits neurotransmitter receptor function, an ATP-gated ion channel interacting with a small hydrophobic pocket on the receptor protein. A 30\% (approximately $100 \mathrm{mM}$ ) ethanol solution is employed since the range of concentrations producing a nervous signal inhibition is $80-120 \mathrm{mM}$ (higher than $20 \%$ ) [21, 33].

The role of US in MN is not merely restricted to a guiding function. In fact, the evaluation of sonographic
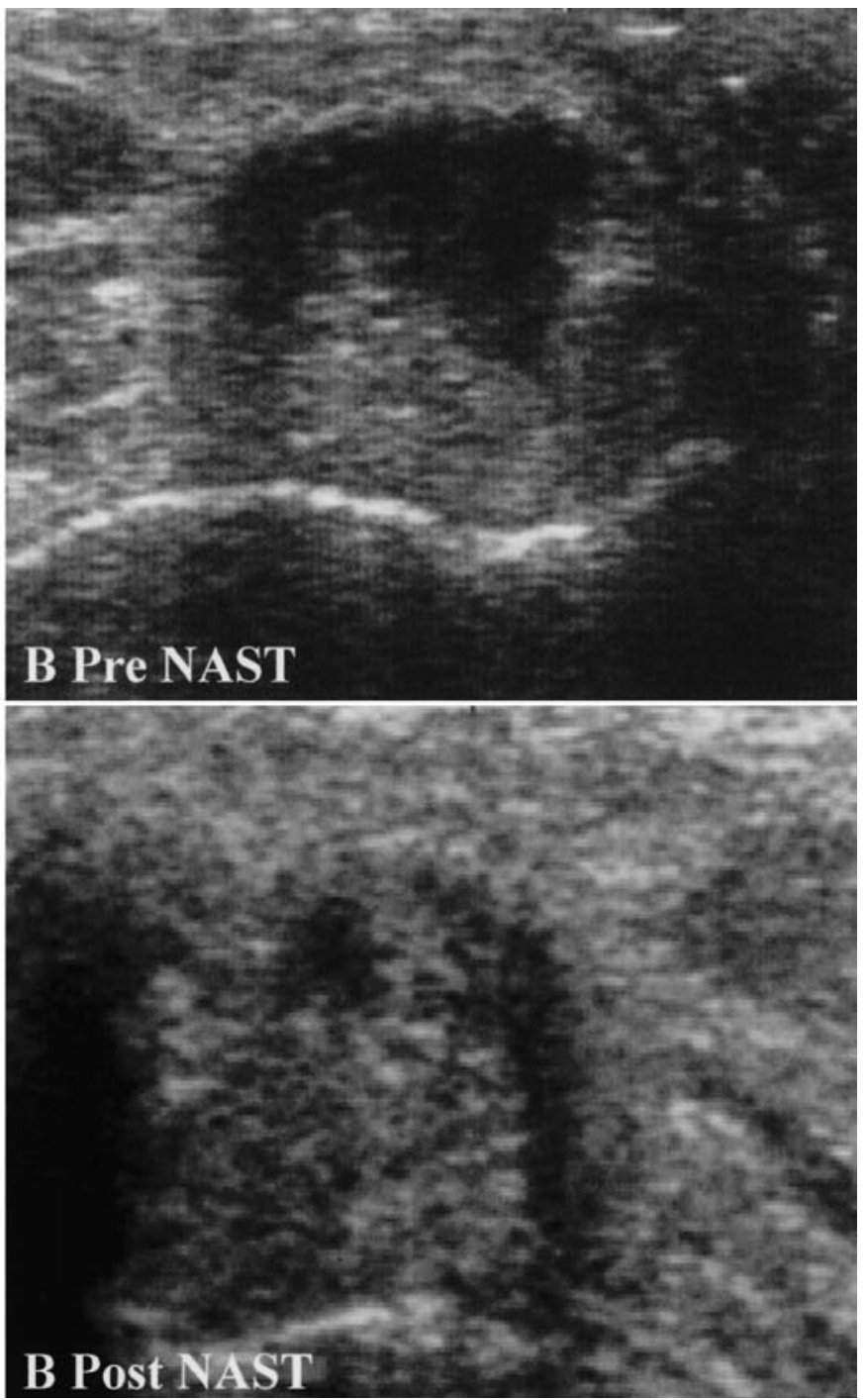

characteristics may have a predictive value for the procedural success. As suggested by the sonographic evaluation of the four lesions undergoing surgery, a type-C echotexture is a negative prognostic factor for the procedural success. In our opinion, this type of echotexture does not allow an optimal alcoholization, although 4 cases are not enough to postulate significant conclusions.

The long-term US evaluation demonstrates how the echotextural change is a significant parameter for the clinical success, whereas mass volume reduction is significant only in terms of an objective grading of the symptomatologic relief. Johnson et al.'s [30] scale, on the other hand, is suitable for the assessment of clinical improvement, although it is a subjective method of evaluation.

Patients undergoing alcohol-sclerosing therapy may be discharged immediately after therapy and can continue their normal daily activities. The pain that may occur 
after injection in the affected area is usually self-limiting and usually does not need any therapy.

Long-term results indicate high rates of clinical success, comparable or greater than those related to other surgical procedure. The role of NAST should be further analyzed in subjects with multiple neuromas and in those cases of surgical failure, after neurectomy, where a new intervention is difficult to propose.

\section{References}

1. Wu KK (1996) Morton's interdigital neuroma: a clinical review of its etiology, treatment, and results. J Foot Ankle Surg 35:112-119

2. Meachim G, Abberton JJ (1971) Histological findings in Morton's metatarsalgia. J Pathol 103:209-217

3. Durlacher L (1845) In: Treatise on corns, bunions, diseases of nails, and the general management of the feet. Simpkin and Marshall, London, p 52

4. Morton TG (1876) A peculiar and painful affection of the fourth metatarsophalangeal articulation. Am J Med Sci 71:37-45

5. Young G, Lindsey J (1993) Etiology of symptomatic recurrent interdigital neuromas. J Am Podiatr Med Assoc 83:255-258

6. Vachon P, Lemay M, Bouchard HL (1991) Pathologic study of Morton's neuroma. Can J Surg 34:356-358

7. Ha'Eri GB, Fornasier VL, Schatzker J (1979) Morton's neuroma: pathogenesis and ultrastructure. Clin Orthop 141:256-259

8. Tate RO, Rusin JJ (1978) Morton's neuroma: its ultrastructural anatomy and biomechanical etiology. J Am Podiatry Assoc 68:797-807

9. Quinn TJ, Jacobson JA, Craig JG, van Holsbeeck MT (2000) Sonography of Morton's neuromas. Am J Roentgenol 174:1723-1728

10. Morscher E, Ulrich J, Dick W (2000) Morton's intermetatarsal neuroma: morphology and histological substrate. Foot Ankle Int 21:558-562

11. Murphey MD, Smith WS, Smith SE, Kransdorf MJ, Temple HT (1999) From the archives of the AFIP. Imaging of musculoskeletal neurogenic tumors: radiologic-pathologic correlation. Radiographics 19:1253-1280
12. Graham CE, Graham DM (1984) Morton's neuroma: a microscopic evaluation. Foot Ankle 5:150-153

13. Bourke G, Owen J, Machet D (1994) Histological comparison of the third interdigital nerve in patients with Morton's metatarsalgia and control patients. Aust N Z J Surg 64:421-424

14. Bencardino J, Rosenberg ZS, Beltran J, Liu X, Marty-Delfaut E (2000) Morton's neuroma: Is it always symptomatic? Am J Roentgenol 175:649-653

15. Mendicino SS, Rockett MS (1979) Morton's neuroma: update on diagnosis and imaging. Clin Podiatr Med Surg 14:303-311

16. Okafor B, Shergill G, Angel J (1997) Treatment of Morton's neuroma by neurolysis. Foot Ankle Int 18:284-287

17. Shapiro PP, Shapiro SL (1995) Sonographic evaluation of interdigital neuromas. Foot Ankle Int 16:604-606

18. Milgram JE (1993) Design and use of pads and strappings for office relief of the painful foot. In: Kiene RH, Johnson KA (eds) Symposium of the foot and ankle. Mosby, St. Louis, pp 95-101

19. Goslin R, Tollafield DR, Rome K (1997) Mechanical therapeutics in the clinic. In: Tollafield DR, Merriman LM (eds) Clinical skills in treating the foot, vol 11. Churchill Livingstone, London, pp187-216

20. Tollafield DR, Pratt DJ, Rome K (1997) Prescription orthoses. In: Tollafield DR, Merriman LM (eds) Clinical skills in treating the foot, vol 11. Churchill Livingstone, London, pp 217-247

21. Dockery GL (1999) The treatment of intermetatarsal neuromas with $4 \%$ alcohol sclerosing injections. J Foot Ankle Surg 6:403-408

22. Masala S, Fanucci E, Ronconi P, Taormina P, Romagnoli A, Simonetti G (2001) Treatment of intermetatarsal neuromas with alcohol injection under US guide. Radiol Med 102:370-373
23. Frisca DA, Strom DE, Parr JW, Saltzman CL, Johnson KA (1991) Surgical treatment for primary interdigital neuroma. Orthopedics 14:669-672

24. Keh RA, Ballew KK, Higgins KR, Odom R, Harkless LB (1992) Longterm follow-up of Morton's neuroma. J Foot Surg 31:93-95

25. Wilson S, Kuwada GT (1995) Retrospective study of the use of a plantar transverse incision vs a dorsal incision for excision of neuroma. J Foot Ankle Surg 34:537-546

26. Gudas C, Mattana G (1986) Retrospective analysis of intermetatarsal neuroma excision with preservation of the transverse metatarsal ligament. J Foot Surg 25:459-463

27. Diebold PF, Daum B, Dang-Vu V, Litchinko M (1996) True epineural neurolysis in Morton's neuroma: a 5-year follow up. Orthopedics 19:397-400

28. Price PD, Miller G (1992) Internal neurolysis. J Foot Surg 31:250-259

29. Mulder JD (1951) The causative mechanism in Morton's metatarsalgia. J Bone Joint Surg 33B:94-95

30. Johnson JE, Johnson KA, Unni KK (1988) Persistent excision on an interdigital neuroma. J Bone Joint Surg 10A:651-657

31. Barrett SL, Pignetti TT (1994) Endoscopic decompression for intermetatarsal nerve entrapment: the EDIN technique: preliminary study with cadaveric specimens; early clinical results. J Foot Ankle Surg 33:503-508

32. Hodor L, Barkal K, Hatch-Fox LD (1997) Cryogenic denervation of the intermetatarsal space neuroma. J Foot Ankle Surg 36:311-314

33. Rengachary SS, Watanabe IS, Singer P, Bopp WJ (1983) Effect of glycerolon peripheral nerve: an experimental study. Neurosurgery 13:681-688 